\title{
Fractal curves and complexity
}

\author{
JAMES E. CUTTING and JEFFREY J. GARVIN \\ Cornell University, Ithaca, New York
}

\begin{abstract}
Fractal curves were generated on square initiators and rated in terms of complexity by eight viewers. The stimuli differed in fractional dimension, recursion, and number of segments in their generators. Across six stimulus sets, recursion accounted for most of the variance in complexity judgments, but among stimuli with the most recursive depth, fractal dimension was a respectable predictor. Six variables from previous psychophysical literature known to effect complexity judgments were compared with these fractal variables: symmetry, moments of spatial distribution, angular variance, number of sides, $P^{2} / A$, and Leeuwenberg codes. The latter three provided reliable predictive value and were highly correlated with recursive depth, fractal dimension, and number of segments in the generator, respectively. Thus, the measures from the previous literature and those of fractal parameters provide equal predictive value in judgments of these stimuli.
\end{abstract}

Fractals are mathematical objects that have recently captured the imaginations of artists, computer graphics engineers, and psychologists. Synthesized and popularized by Mandelbrot $(1977,1983)$, with ever-widening appeal (e.g., Peitgen \& Richter, 1986), fractals have many curious and fascinating properties. Consider four.

First, and by neology, fractals are fractional-dimensioned objects. That is, whereas we are most accustomed to thinking in terms of objects with ordinal dimensions of $0,1,2$, and 3-for points, lines, planes, and solids, respectively-fractals allow dimensional values to be anywhere among the positive real numbers. Thus, one can generate a curve with a fractal dimension of, say, 1.67 or a surface with 2.33 dimensions. In this paper we concentrate on fractals with between 1.0 and 2.0 dimensions, which are called fractal curves.

Second, fractals are synthesized by considering two entities: generators and initiators. For fractals with between 1.0 and 2.0 dimensions, one considers the relation between length along the curve and the distance between its endpoints. The length along the curve is a property of the generator; the distance between endpoints is a property of the initiator. The simplest case is of an initiator of two points and a straight-line generator that connects them. This object, not surprisingly, has a fractional dimension of 1.0 ; it is a line. But consider next a jagged line that connects these two points indirectly, meandering through two-dimensional space as shown in the left panel of Figure 1. In this case, because the segments within the generator are of equal unit lengths, one can

This work was supported by Grant MH37467 from the National Institute of Mental Health to the first author, and was made possible by Lynn Cooper and the Department of Psychology of the University of Arizona, during the first author's sabbatical from Cornell University. We thank Joseph J. Stevens for discussions of complexity. J. Garvin is currently at NEC Corporation in Tokyo. Requests for reprints should be sent to J. Cutting, Department of Psychology, Uris Hall, Cornell University, Ithaca, NY 14853-7601. determine the fractional dimension by dividing the logarithm of the number of unit lengths in the generator by the logarithm of the number of unit lengths across the initiator. Since there are five segments in this generator and three unit lengths across the initiator, the fractional dimension is $\log (5) / \log (3)$, or about 1.47 .

Third, all true fractals possess the property of recursion. In this context, recursion means successive reapplication of the generator to its subsegments. Thus, the first recursion makes each original segment a new initiator; the second recursion, in turn, makes each new subsegment an initiator, and so on. The first four recursions of this fractal are shown in the left panel of Figure 2. True fractals have infinite recursive depth. Recursion leads to the most important property of fractals: they are selfsimilar at different scales. Since this idea can be a bit complicated, consider a generator taken from Mandelbrot (1983) and shown in the middle panel of Figure 1. This one has nine-unit segments, covering an initiator of three units; the first four recursions are shown in the middle panel of Figure 2. Infinite recursion makes the space look blacker and blacker, because we have the oddity of infinitely thin lines packing space at an exponential rate. The pattern begins to look like a solid sheet, but every subpart of it looks like every part-it is self-similar. Fortunately, the fractal dimension of this object is $\log (9) /$ $\log (3)$, or 2 .

As an aside, let us note two things: (1) The infinite recursive depth creates a tension between fractals as mathematical objects and fractals as psychological objects and as measured and applied to the real world. Typically, natural objects, if they can be said to have any fractal dimension at all, have a self-similarity only to about three or four levels of recursion. The coastlines used as a didactic tool by Mandelbrot $(1977,1983)$ would seem to be exceptions that allow deeper recursions. (2) Fractal curves should be self-avoiding. The fractals just discussed have such a feature; that is, segment lines never cross each other regardless of recursive depth. 


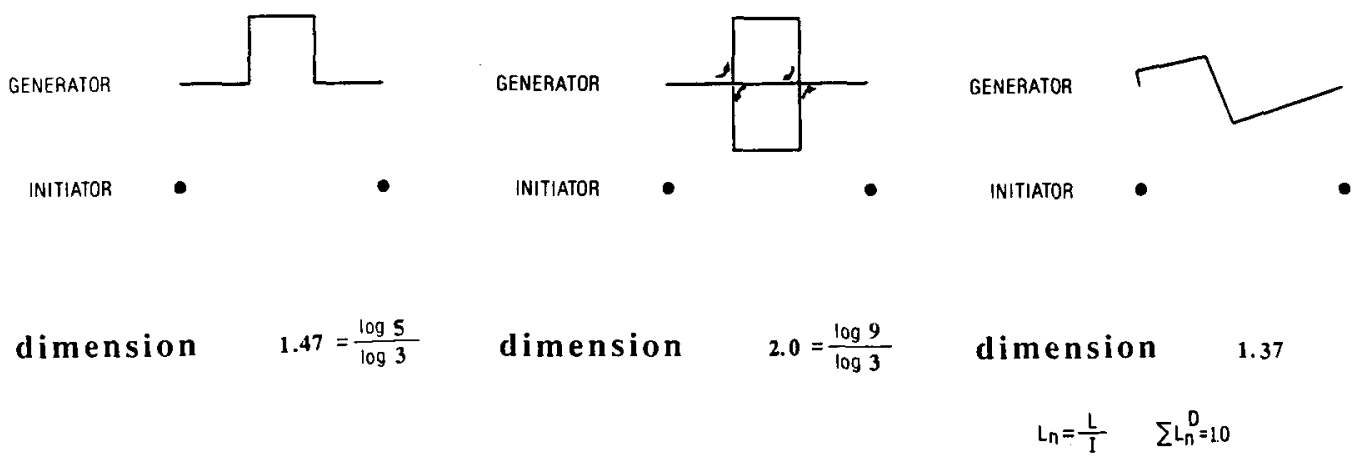

Figure 1. Three fractal generators and their simple initiators. The first two have constant segment length; the third has variable segment length.

A fourth property of fractals, if we confine ourselves to fractal curves, is that they come in three kinds. We have already discussed the first, which is made up of constant-length segments. The second has variable-length segments. These are generated as shown in the last panel of Figure 1; they, too, are recursive and should be selfavoiding. To determine dimensionality, however, one must take a slightly different approach. The length of each segment must be normalized, dividing its length by the distance across the initiator. Then these are summed, each taken to the $D$ th power, where $D$ is the fractional dimension of the curve (Mandelbrot, 1977). Their sum must equal 1.0. We use these variable-segment-length fractals in the experiment discussed here. As an aside, notice in Figure 2 that this particular object is not a true fractal, because it is not self-avoiding beyond the second recursion. In general, making variable-length multi-segment generators self-avoid can be quite difficult.

Before we discuss our experiment, consider the third kind of fractal. These, too, have variable-length segments, but they are no longer required to replicate themselves exactly with each recursion. The only property they need is that the sum, upon each recursion, of the normalized segment lengths taken to the $D$ th power be equal to 1.0.

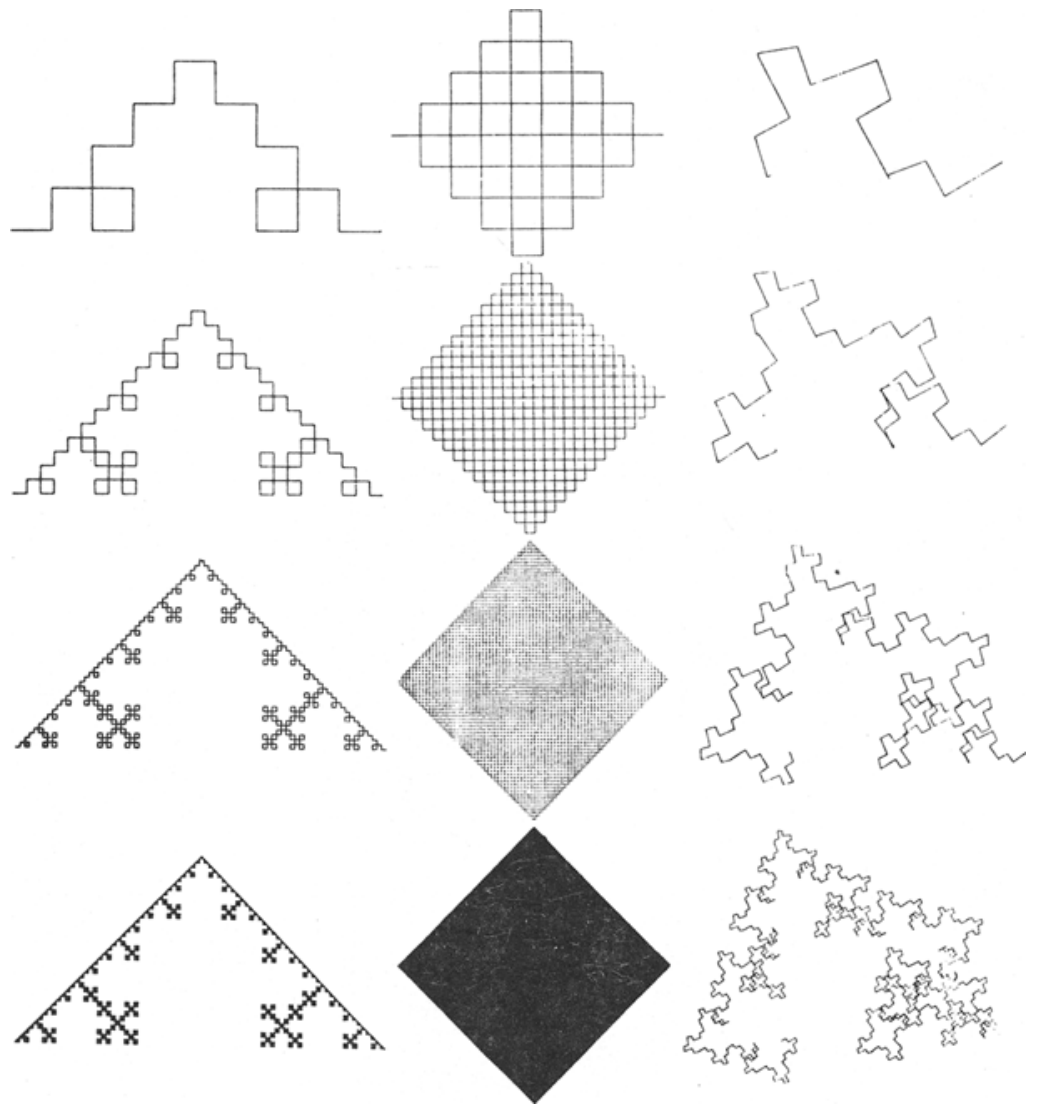

Figure 2. The same three fractal curves from Figure 1, taken to their first, second, third, and fourth recursions. 
At dimensions appraching 2.0 , making these figures selfavoid is also very difficult. Note that whereas this third type of fractal is only self-similar at different scales, the first two types were actually self-identical. This third type is called a brown fractal, because it can be used to mimic Brownian motion; it is also Mandelbrot's $(1977,1983)$ central contribution, other than synthesis and popularization of a disparate literature, to this mathematical domain.

The computer graphics community has been so excited about fractals because brown fractals of about 2.1 dimensions can look very much like the Appalachians, and those of about 2.35 dimensions can look like the Alps. In other words, they are good objects for synthesizing certain kinds of natural-looking environments. My interest in fractals, however, other than their aesthetic appeal, stems from the work of Pentland (1984) and explores a different direction, that of conceptual backfill.

Pentland $(1984,1986)$ performed two psychophysical studies of fractals. In the first study, he generated Brownian movements along a line, with fractal dimensions between 1.0 and 2.0, and had people judge their roughness.
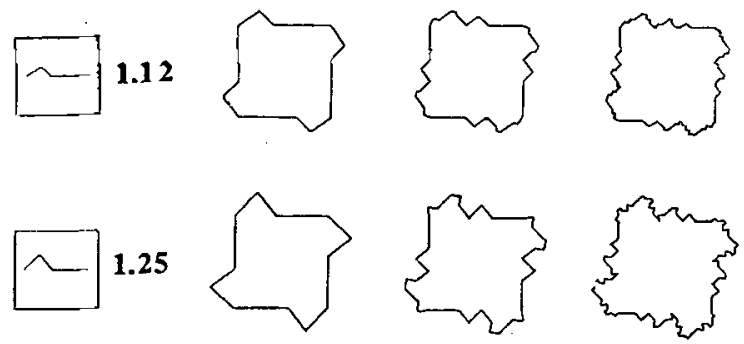

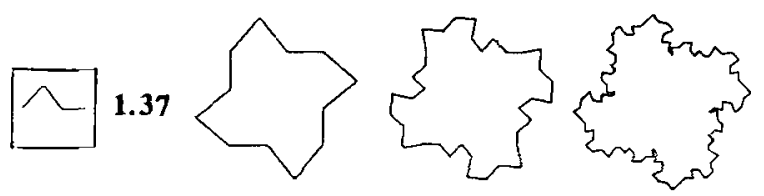

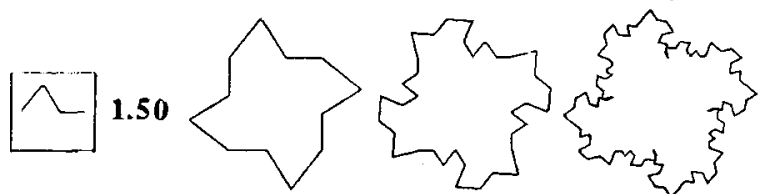

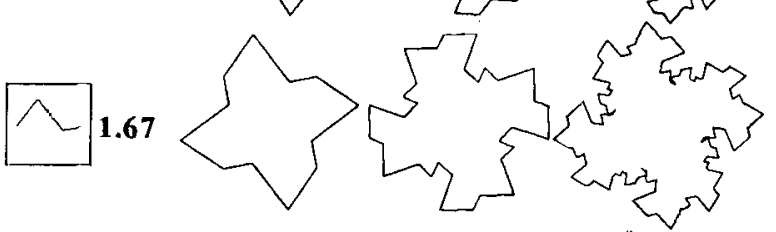

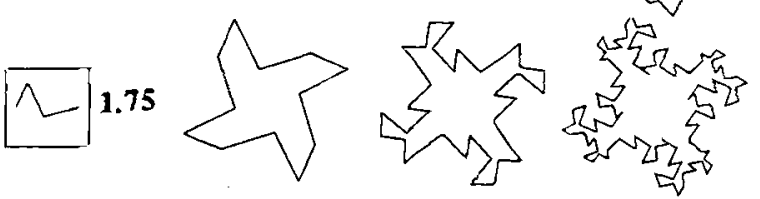

Figure 3. Three-segment variable-length fractal generators and 18 stimuli generated from them, with six different fractal dimensions and three recursion depths.
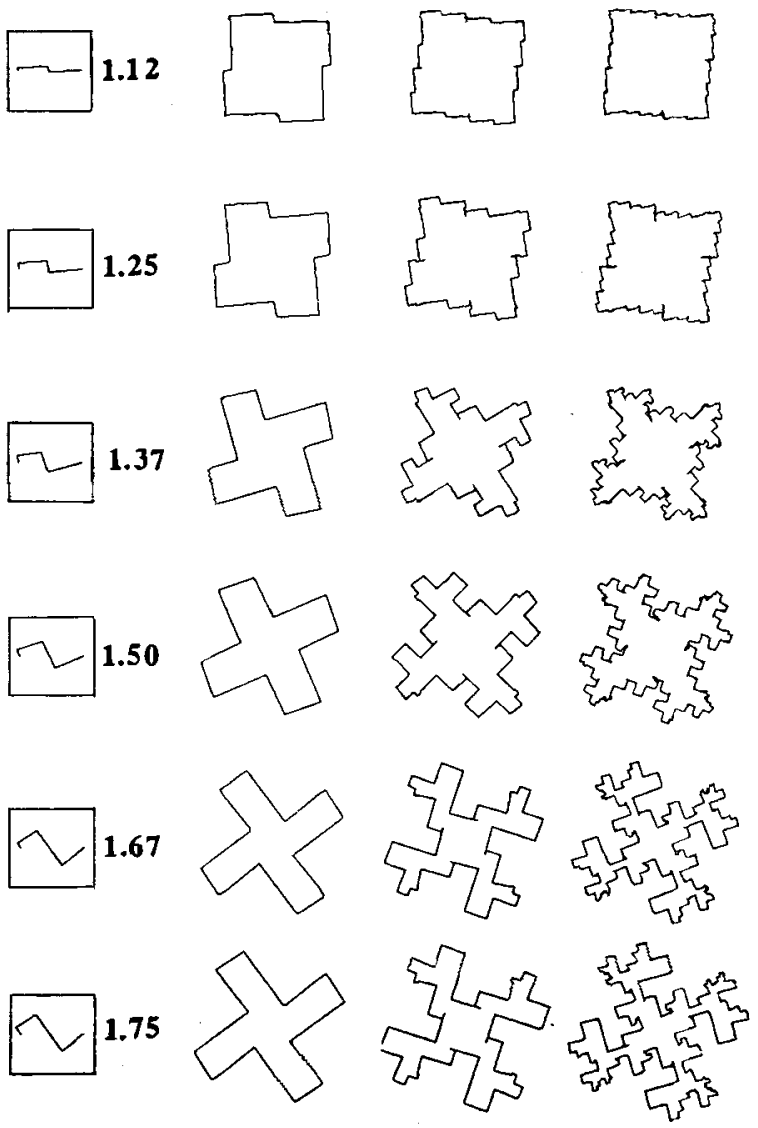

Figure 4. Four-segment variable-Jength fractal generators and their stimuli.

In the second study, he quantitized textures from Brodatz (1966), determined their fractal dimension (here between 2.0 and 3.0 dimensions because they tended to fill space, rather than to fill a plane) with some assumptions about light source and shading, had subjects imagine that they were rubbing the surfaces of these textures, and had them rate the textures' roughnesses. In both studies the correlation between roughness and fractal dimension was about .90 or better. Marshak (1986) found similar, though somewhat less striking, results. Pentland (1986) went on to do other analyses of fractals, including their relation to spatial-frequency analysis under certain constrained conditions (see also Kube \& Pentland, 1986).

It seemed to us that roughness was a psychological dimension that might be highly correlated with complexity, and that complexity had a decades-old tradition in visual psychophysics that might be profitably updated in the context of fractals. In essence, then, we decided to generate some fractal curves with certain properties, have people judge their complexity, and compare the dimensions of the fractals with the stimulus dimensions used in the previous literature. 


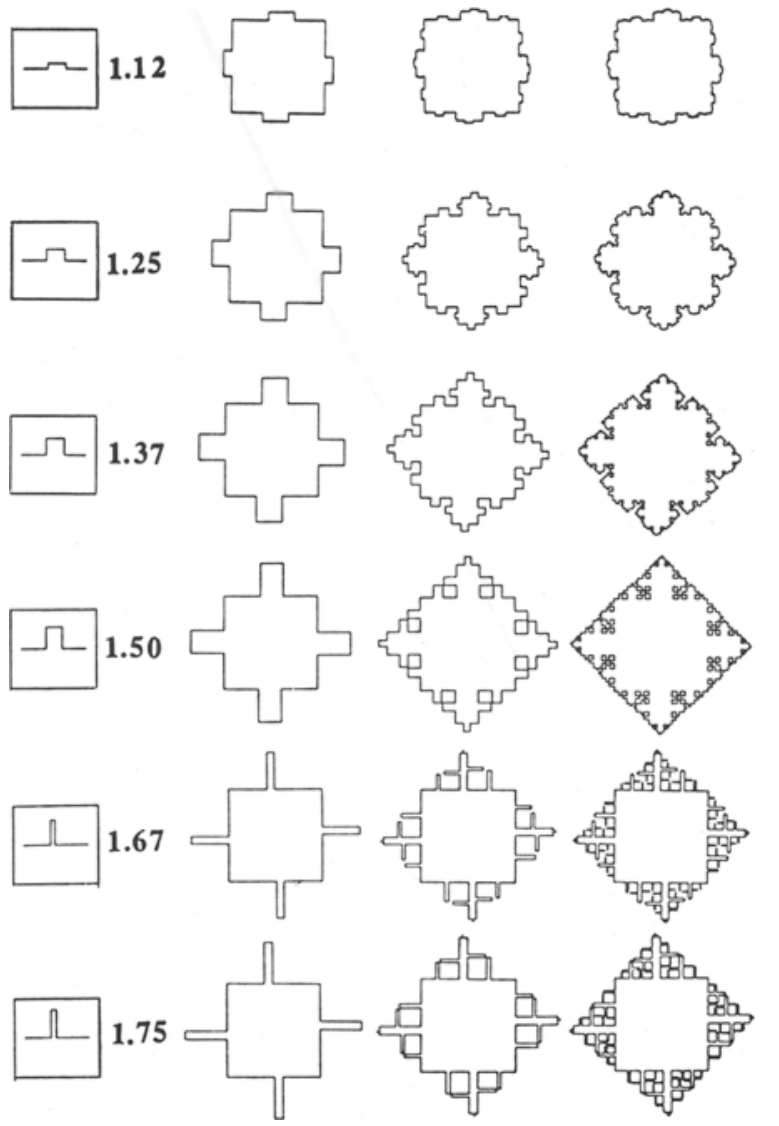

Figure 5. Five-segment variable-length fractal generators and their stimuli.

\section{METHOD}

\section{Stimuli and Apparatus}

We generated six sets of fractals on an Apple Macintosh microcomputer: two with three segments in the generator, two with four segments, and two with five segments. No sets with two-segment generators were used, since they do not allow the dynamic range of fractal dimension used here, and none were used with more than five segments for the pragmatic reason that computation times go up exponentially with number of segments in the generator. Two sets each were generated with three, four, and five segments to enhance generality.

Each set had segment lengths and/or angular intersections adjusted so that six fractal objects could be generated, with dimensions of $1.125,1.250,1.375,1.500,1.675$, and 1.750. Each of these was then generated at three recursion depths: $\mathbf{0}$ (using only the generator), 1 , and 2 . We did not go beyond these levels for three reasons: (1) we were already taxing the resolution of the display and printing system at level two; (2) at the next level, we would begin to tax the human visual system; and (3) all of these stimuli self-avoid, and generating variable-segment-length fractals that were self-avoiding would be much more difficult at the next level of recursion.

These objects were placed on two initiators of four dots, one in the shape of a square, and the other in the shape of a diamond. These were used because the graphics on a Macintosh are rasterscan and, thus, are subject to spatial aliasing (the "jaggies," due to discrete sampling). The use of two types of displays, one rotated $45^{\circ}$ to the other, allowed for some counterbalancing of aliasing artifacts. The 216 stimuli were then laser-printed, photocopy- reduced, and mounted in scrambled order in two notebooks. Figures 3, 4, and 5 show 54 of the stimuli, 18 from each of three sets. Each figure shows the generators on the left, and the three stimuli from each generator on the right. These represent the three levels of recursion that we used. The first set of stimuli has three segments in its generator, the second has four segments, and the third has five segments. The 18 generators for the other 54 stimuli are shown in Figure 6.

\section{Subjects}

Eight adults participated individually in the experiment. Each subject was a member of the Psychology Department community of the University of Arizona, and each volunteered. Only 2 subjects had a prior understanding of fractals.

\section{Procedure}

Before beginning, each participant perused the two notebooks to get a sense of the range of stimuli. He or she then rated each fractal on a 1-to-10 scale as to how complex it appeared, with 10 representing the most complex stimuli. Half of the subjects began with one notebook, half with the other. The task was self-paced and took 15 to $40 \mathrm{~min}$ to complete.

\section{RESULTS AND DISCUSSION}

Mean intercorrelation among participants' complexity ratings was quite high $(r=.80)$ with a modest range ( $r s=.64$ to .88 , all $p s<.001$ ). Intersubject reliability was thus gratifyingly high and sufficient to suggest that
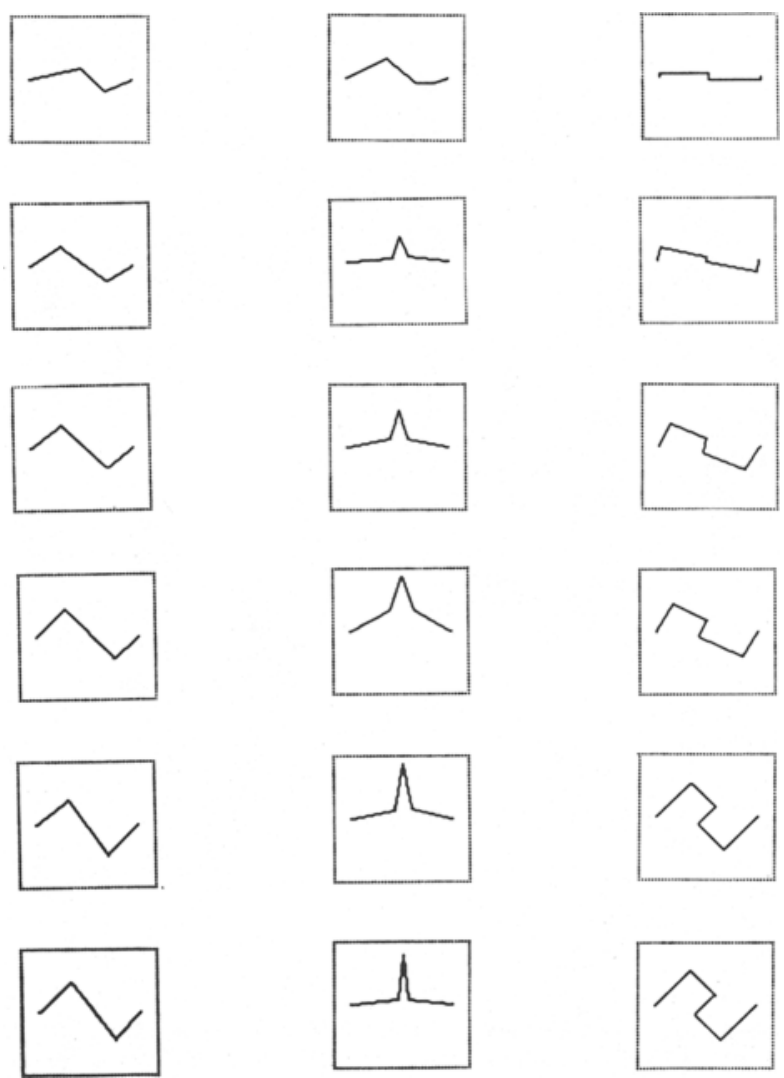

Figure 6. The other 18 fractal generators with three, four, and five segments. 
Table 1

Three Stimulus Properties of Fractal Curves and Their Correlation with the Complexity Judgments

\begin{tabular}{lcc}
\hline & All Data & 2nd Recursion \\
\hline Fractal Dimension & .28 & .68 \\
Number of Segments & .20 & .47 \\
Recursion Depth & .86 & - \\
$R$ & .93 & .82 \\
\hline
\end{tabular}

Note-All correlations are significant at $p<.0001$.

there were no noteworthy individual differences. Further analyses were performed on means, and the general results are shown in Table 1.

The three orthogonal stimulus variables of fractal dimension, recursion depth, and number of segments in the generator were of greatest interest and were the only ones that contributed to the prediction. (Two other properties, type of generator within a particular number of segments and orientation of the initiator, did not reliably correlate with complexity judgments, $r s=.01$ and .02 , respectively.) For the three principal dimensions taken together, the multiple correlation was quite high $[R=.93, F(3,212)$ $=459.3, p<.0001]$, accounting for $86 \%$ of the variance in the data. Beta weights, or in this case simple correlations, had an interesting pattern: They were relatively modest for the number of segments in the generator $[r=$ $.20, t(212)=8.06, p<.0001]$, which might be expected since it is a variable that is not truly part of the measurement of fractals, but the simple correlations were also modest for fractal dimension itself $[r=.28, t(212)=$ $11.18, p<.0001]$. Much more of the variance was accounted for by recursion depth $[r=.86, t(212)=34.5$, $p<.001]$.

The low correlation for fractal dimension might be regarded as embarrassing for our attempt to relate fractals and complexity, but one must remember that true fractals have infinite recursion depth. Thus, technically speaking, none of these stimuli were fractals; they were only fractal-like. The most fractal-like curves were the $\mathbf{7 2}$ stimuli at the second recursion depth, so consider these alone. For them, the fractal-dimension and segmentnumber variables provided a multiple correlation modestly lower than that for all variables and all the data $[R=.82$, $F(2,69)=35.1, p<.0001]$, and fractal dimension did much better as a predictor of complexity judgments $[r=$ $.68, t(69)=9.8, p<.0001]$. The number of segments in the generator was still reliable as a predictor of the data $[r=.47, t(69)=6.7, p<.0001]$. Since number of segments was irrelevant to the computation of fractal dimension, one can say that there was more to these judgment data than could be captured by fractals.

Thus, as an internal summary, true fractal properties (fractal dimension and recursion depth) do quite well in predicting judgments of complexity, but the number of segments in the generator (which does not manifestly effect fractal dimension) adds predictive value as well.

The second focus of this experiment was to compare these synthesis variables with some of those we thought important from the previous literature on complexity judgments of visual forms. We considered six: symmetry (e.g., Brown, Hitchcock, \& Michels, 1962; Zusne \& Michels, 1962), moments of spatial distribution (e.g., Alt, 1962; Knoll \& Stenson, 1968; Sutherland, 1957), angular variability (e.g., Attneave, 1957), the numbers of sides (e.g., Casperson, 1950), the relations of the perimeter length to the area (e.g., Attneave, 1957), and the structural codes from Leeuwenberg $(1971,1978)$. Zusne (1970) provided an overview of all of these but the last.

The overall patterns of prediction are shown in Table 2 . The first three variables offered little for these stimuli. As it turns out, symmetry was irrelevant to these stimuli: All stimuli were rotationally symmetric to the same degree, given that their initiators were squares or diamonds. Second, complexity judgments did not vary with the first through fourth moments of spatial distribution: the mean, variance, skewness, and kurtosis of a onedimensional distribution. The highest correlation for any of these at any rotation was poor $(r=.09)$. The third variable, angular variability (taken to be the variance of the angles of the stimuli), also correlated poorly with the data $(r=.11)$.

The latter three variables, on the other hand, were quite potent predictors of complexity judgments. The fourth variable was simply a measure of the number of sides to the stimulus. True fractals, because of their infinite recursive depth, have an infinite number of sides; but the stimuli used here, of course, did not. The number of sides correlated with the data moderately well $(r=.72)$, but a better measure was the logarithm of that number $(r=.88)$. The fifth variable was the perimeter squared over the area, $P^{2} / A$, which correlated with the data reasonably well $(r=$ .58 ) but, because of the exponential increase in perimeters with recursive depth, the logarithm of $P^{2} / A$ was a better measure $(r=.84)$. Finally, a reasonable set of structural information codes from Leeuwenberg (1978) correlated moderately with the data $(r=.56)$. Here, all stimuli coded the segment length as two variables (length and angle) and recursion as one (see Leeuwenberg, 1978). This might be considered an embarrassment to a coding the-

Table 2

Nonfractal Dimensions and Their Correlations with Complexity Judgments and Synthesis Parameters

\begin{tabular}{|c|c|c|}
\hline & $\begin{array}{l}\text { Correlation with } \\
\text { Complexity }\end{array}$ & $\begin{array}{c}\text { Correlation with } \\
\text { Synthesis Parameters }\end{array}$ \\
\hline Symmetry & - & - \\
\hline $\begin{array}{l}\text { Moments of } \\
\text { Spatial Distribution }\end{array}$ & $<.10$ & - \\
\hline Angular Variance & .11 & - \\
\hline $\begin{array}{l}\text { Number of Sides } \\
\log (\# \text { sides })\end{array}$ & $\begin{array}{l}.72 \\
.88\end{array}$ & .92 with $\overline{R e c u r s i o n}$ \\
\hline $\begin{array}{l}\text { Perimeter Squared } \\
\text { Over Area } \\
\log \left(P^{2} / A\right)\end{array}$ & $\begin{array}{l}.58 \\
.84\end{array}$ & $\begin{array}{l}.82 \text { with Fractal } \\
\text { Dimension }\end{array}$ \\
\hline Leeuwenberg Codes & .56 & $\begin{array}{l}.89 \text { with Number of } \\
\text { Segments }\end{array}$ \\
\hline
\end{tabular}


ory that centers on stimulus variables of generation but, to be fair, Leeuwenberg never suggested that complexity was captured by his coding scheme, only simplicity.

When the last five variables were entered into a regression equation no more of the variance was accounted for than was with only the three fractal variables $(R=.94)$. In fact, almost all of the variance was accounted for by the last three, and each of these correlated highly with one of the variables used in the experiment: logarithm of the number of sides with recursion $(r=.92)$, logarithm of $P^{2} / A$ with fractal dimension $(r=.82)$, and Leeuwenberg codes with number of segments $(r=.89)$. Thus, one can say that fractal parameters and those measures from the older literature on figural complexity assessed similar stimulus properties and accounted for the data equally well.

In summary, four things can be said. First, for these variable-length fractal objects, the dimensions of synthesis-fractal dimension, number of segments, and recursion depth - together provide a good prediction of complexity judgments. Second, the number of segments in the fractal generator accounted for some of the variance in the data in places that one might not have expected. Third, fractal dimension and recursive depth contributed substantially, and fractal dimension was the most powerful variable among the most recursive stimuli. Fourth, the variance in the data was accounted for equally well by variables from the previous complexity literature. Those that were reliable predictors were $P^{2} / A$, the number of sides, and a Leeuwenberg coding scheme.

\section{REFERENCES}

ALT, F. L. (1962). Digital pattern recognition by moments. In G. L. Fischer et al. (Eds.), Optical character recognition (pp. 153-179). Washington, DC: Spartan.
Attneave, F. (1957). Physical determinants of the judged complexity of shapes. Journal of Experimental Psychology, 61, 183-193.

Brodatz, P. (1966). Textures. New York: Dover.

Brown, D. R., Hitchcock, L., \& Mrchels, K. M. (1962). Quantitative studies in form perception: An evaluation of the role of selected stimulus parameters in the visual discrimination performance of human subjects. Perceptual \& Motor Skills, 14, 519-529.

Casperson, R. C. (1950). The visual discrimination of geometric form. Journal of Experimental Psychology, 40, 668-686.

KNOLL, R. L., \& STENSON, H. H. (1968). A computer program to generate and measure random forms. Perception \& Psychophysics, 3, 311-316.

Kube, P., \& Pentland, A. (1986). On the imaging of fractal surfaces (SRI Technical Note \#390). Palo Alto, CA: SRI.

LEEUWENBERG, E. (1971). A perceptual coding language for visual and auditory patterns. American Journal of Psychology, 84, 307-347.

LEEUWENBERG, E. (1978). Quantification of certain visual pattern properties: Salience, transparency, similarity. In E. Leeuwenberg \& H. Buffart (Eds.), Formal theories of visual perception (pp. 277-298). Chichester, England: Wiley.

MANDELBRot, B. B. (1977). Fractals: Form, chance, and dimension. San Francisco: Freeman.

MANDELBROT, B. B. (1983). The fractal geometry of nature. San Francisco: Freeman.

MARSHAK, F. M. (1986). Stochastic models in the visual perception of natural surfaces. Unpublished manuscript.

Pertgen, H.-O., \& Richter, P. H. (1986). The beauty of fractals. Berlin: Springer-Verlag.

Pentland, A. P. (1984). Fractal-based description of natural scenes. IEEE Pattern Analysis and Machine Intelligence, PAMI-6, 661-674.

Pentland, A. P. (1986, June). On perceiving 3-D shape and texture. Paper presented at the Symposium on Computational Models in Human Vision, Rochester, NY.

SUTHERLAND, N. S. (1957). Visual discrimination of orientation and shape by Octopus. Nature, 179, 11-13.

Zusne, L. (1970). Visual perception of form. New York: Academic Press.

ZuSNE, L., Michels, K. M. (1962). Geometricity of visual form. Perceptual \& Motor Skills, 15, 55-58.

(Manuscript received November 10, 1986; revision accepted for publication April 13, 1987.) 